\title{
Reservoir characterization based on seismic spectral variations
}

\author{
Yanghua Wang ${ }^{1}$
}

\begin{abstract}
The seismic frequency spectrum provides a useful source of information for reservoir characterization. For a seismic profile presented in the time-space domain, a vector of the frequency spectrum can be generated at every sampling point. Because the spectrum vectors at different time-space locations have different variation features, I attempt for the first time to exploit the variation pattern of the frequency spectrum for reservoir characterization, and test this innovative technology in prediction of coalbed methane (CBM) reservoirs. The prediction process implicitly takes account of the CBM reservoir factors (such as viscosity, elasticity, cleat system, wave interference within a coal seam, etc.) that affect the frequency spectrum, but strong amplitudes in seismic reflections do not necessarily show any influence in clustering analysis of spectral variation patterns. By calibrating these variation patterns quantitatively with CBM productions in well locations, we are able to characterize the spatial distribution of potential reservoirs.
\end{abstract}

\section{INTRODUCTION}

In this study, I propose to exploit the variation patterns of seismic frequency spectra for characterizing the spatial distribution of potential reservoirs. Considering, for example, a 2D seismic profile in the time-space domain, a vector of the frequency spectrum can be generated at every sample point. Such frequency spectral vectors at different time-space positions have different variation patterns. If using different colors to present the time-space points with different variation patterns, one can create a $2 \mathrm{D}$ color image. This colorful $2 \mathrm{D}$ picture has the potential to highlight the reservoir anomalies. As a demonstration, I apply this technology to predict the spatial distribution of coalbed methane (CBM) reservoirs, which is an important unconventional energy resource (Shuck et al., 1996; Bachu and Michael, 2003; Peng et al., 2006).
The "data" I use in prediction are the frequency spectra. Thus, the first step of the entire procedure is to raise an extra dimension. For a conventional 2D seismic profile in the time-space domain, I generate a 3D data cube in which the third dimension is frequency. This data cube in time-space-frequency domain is often called a time-frequency spectrum. The second step is clustering analysis, which is a dimension-reduction process that reduces the data dimensions from three down to two. The resultant image is presented again in the time-space domain. The third step is characterization which calibrates the indexes of spectral patterns with known CBM production and predicts the CBM spatial distribution.

I generate the time-frequency spectrum using the matching pursuit technique (Wang, 2007, 2010). However, because there is no straightforward relationship between the CBM content and the seismic strength, one cannot make a quantitative characterization of CBM spatial variation based on various types of amplitudes either in the frequency domain or the time domain. Therefore, in this study I propose for the first time to predict the spatial distribution of coal seam and its methane content, based on the variation characteristics of the seismic frequency spectrum.

The essential message here is that it is the spectral variation along the frequency axis, rather than the amplitude of each individual frequency component, that plays a key role in reservoir characterization. A strong reflection in the seismic profile, either in the time or frequency domain, does not necessarily show any influence in spectral-variation-based reservoir characterization.

\section{STUDY AREA}

Coalbed methane used to be a mining hazard, which has now been converted into an environmentally friendly fuel. As a significant energy resource, CBM is cleaner than any other fossil fuel. It has been claimed that the worldwide resources of methane trapped within the cleat and fractured coal seams are greater than the total reserves of all known conventional natural gas fields (Bachu and Michael, 2003).

\footnotetext{
Manuscript received by the Editor 1 September 2011; revised manuscript received 26 May 2012; published online 27 September 2012.

${ }^{1}$ Imperial College London, Centre for Reservoir Geophysics, Department of Earth Science and Engineering, London, U. K. E-mail: yanghua.wang @ imperial ac.uk.

(C) 2012 Society of Exploration Geophysicists. All rights reserved.
} 
In the study area, the Qinshui basin of China, there is a series of coal seams in sedimentary rocks of the $T$ (Taiyuan) formation in the Upper Carboniferous and of the S (Shanxi) formation in Permian. The thickness of the bottom $\mathrm{T}$ formation is $100.9-150.8 \mathrm{~m}$, and the thickness of the top $\mathrm{S}$ formation is $31.5-78.2 \mathrm{~m}$. The total thickness of these two formations is $132.4-229.0 \mathrm{~m}$ with an average $175.3 \mathrm{~m}$. Within these two formations, there are 17 coal seams, in which 1-6 coal beds are in the top $\mathrm{S}$ formation and the rest in the bottom $\mathrm{T}$ formation. The total thickness of coal seams is $14.05 \mathrm{~m}$, which is $8 \%$ of the total sedimentary thickness.

Among these 17 coal seams, there are 5-7 industrially producible coal layers, and two major CBM layers, which are shown as coal seam 15 in the bottom $T$ formation and coal seam 3 in the top $S$ formation. For these two CBM layers, the thickness of each coal bed is very thin, as revealed by drilling information. The thickness of coal seam 15 is between a minimum of $3.0 \mathrm{~m}$ and a maximum of $6.7 \mathrm{~m}$, with a steady average $5.17 \mathrm{~m}$ over the area. The thickness of coal seam 3 is between $0 \mathrm{~m}$ and $3.7 \mathrm{~m}$ with an average $1.08 \mathrm{~m}$. The average thickness of this coal seam is also relatively steady over the area. However, because the CBM reservoir has a strong spatial variation, it is difficult to delineate methane rich sweet spots within coal seams.

Figure 1 displays well-logging data from well $\mathrm{H} 2$ and seismic traces around the well. The two CBM layers are indicated as $\mathrm{R} 3 \mathrm{c}$ and R15c, the top and bottom boundary of formation S in Permian are indicated as R2 and R3, and the top and bottom boundary of formation $\mathrm{T}$ in the Upper Carboniferous are indicated as R3 and $\mathrm{R} 4$. In addition, the top of Ordovician is indicated as R5. The tops of CBM layers R3c and R15c appear as a trough in seismic waveforms. There is shown a good seismic well tie for R15c, but the group of reflections, R2-R3c-R3, in the seismic traces is advanced systematically about a quarter of a wavelet, in comparison with synthetics.

Figure $2 \mathrm{a}$ and $2 \mathrm{~b}$ displays two seismic profiles across the well. The straight red vertical line indicates the well location, and its bottom is the reflection of the target coal seam 15. On these seismic profiles, R3 is a strong reflection separating formation $\mathrm{S}$ from

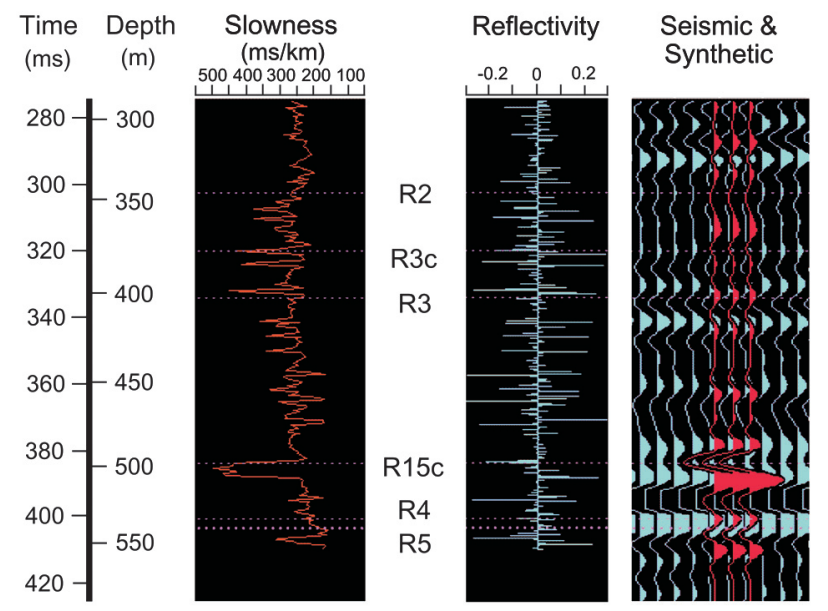

Figure 1. Seismic two-way traveltime, depth, acoustic slowness $(\mathrm{ms} / \mathrm{km})$, seismic reflection index, the reflectivity series, seismic traces (light blue), and the synthetic trace (red) at well location $\mathrm{H} 2$. The top of CBM layers R3c and R15c appears as a trough in seismic waveforms. formation T. It has lateral continuity with a strong peak and two weak troughs, and can be used as a good reference in seismic profiles over the area. Above R3, there is the reflection R3c, which is not only the reflection of coal seam 3 , but also combines reflections
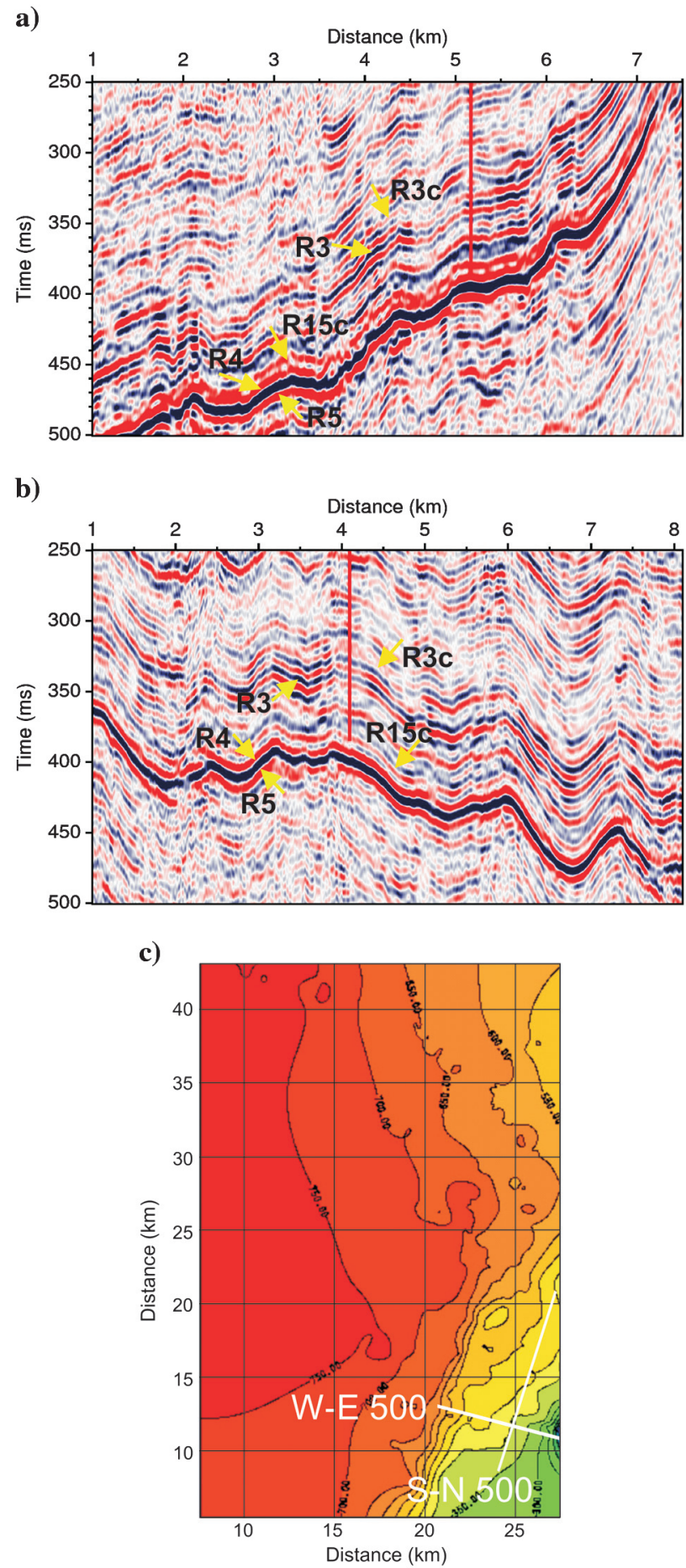

Figure 2. (a and b) Two seismic profiles, W-E 500 and S-N 500, respectively, across well $\mathrm{H} 2$. (c) The contour of reflection time (in $\mathrm{ms}$ ) of R4-R5 (from the bottom of formation T in Upper Carboniferous and the top of Ordovician limestone), which can also reflect the structural variation of the coal seam reflection $\mathrm{R} 15 \mathrm{c}$, on the top of R4-R5. 
of other coal seams within formation S. This reflection is spatially traceable with weak coherence in some places which could reflect the thickness variation of coal seams.

Reflection R4 (from the bottom of formation $\mathrm{T}$ in the Upper Carboniferous) and R5 (from the top of the Ordovician limestone) together have strong amplitudes and very good lateral continuity, and can be traced very easily across the entire study area. Just above $\mathrm{R} 4-\mathrm{R} 5, \mathrm{R} 15 \mathrm{c}$ is the reflection from coal seam 15 , the main target of our CBM study. Due to the steady sedimental environment, this coalbed reflection with good lateral continuation can be easily traced in seismic profiles.

Figure 2c displays the contour of reflection time (in ms) of R4R5. This time contour can also show the structural variation of coal seam 15, which is reflection R15c, about $15 \mathrm{~ms}$ above R4-R5.

\section{THE TIME-FREQUENCY SPECTRUM}

"Thin bed" is a relative concept depending upon the actual seismic resolution. If the thickness of a layer is less than the seismic resolution, it is a thin bed. For the detection of a thin bed that is beyond the seismic resolution limit in the time domain, one needs to exploit the dynamic characteristics of seismic waves, including the frequency content and the spectral variation pattern. These characteristics may implicitly reflect the effects of lithology, mineral content, fracture, and thin-bed thickness, etc.

I use a matching pursuit method to calculate the time-frequency spectrum (Wang, 2010). Matching pursuit is a wavelet decomposition method that extracts wavelets from a seismic trace and presents them in the time-frequency domain. It has been developed to overcome the limits in two conventional methods: windowed Fourier transform (or Gabor transform), and the wavelet transform. In windowed Fourier transform with fixed window size, the length of extracted wavelet is a constant proportional to the window size. Thus, it cannot accurately show the actual scale variation in the signal. In contrast, the length of wavelet in a wavelet transform is variable, but it is strictly inverse-proportional to the dominant frequency (Mallat, 2009). Therefore, neither of these two popular methods can accurately estimate the frequency content, especially high frequencies, from field seismic data. Matching pursuit is an adaptive decomposition method, and the extracted wavelets will match the time and frequency structure of field seismic signal (Mallat and Zhang, 1993).

Most wavelet decomposition methods use a symmetric, noncausal wavelet such as the Ricker wavelet as the basic wavelet (atom). Matching pursuit (Wang, 2007) adopts the Morlet wavelet as the atom which can represent the attenuation and absorption effect of the seismic wave propagating through the subsurface media (Morlet et al., 1982a, 1982b). In addition, this implementation introduces a variable wavelet scale to increase accuracy in the wavelet decomposition.

The question is whether we can find out spectral anomalies related to CBM enrichment, based on the analysis of the frequency spectrum at each time-space position. Figure 3 displays a timefrequency spectrum around the well location, along profile W-E 500. For strong reflection R4-R5, the amplitudes of different frequencies have a good lateral continuation. However, the strength of the coal seam reflection R15c immediately above R4-R5 is much
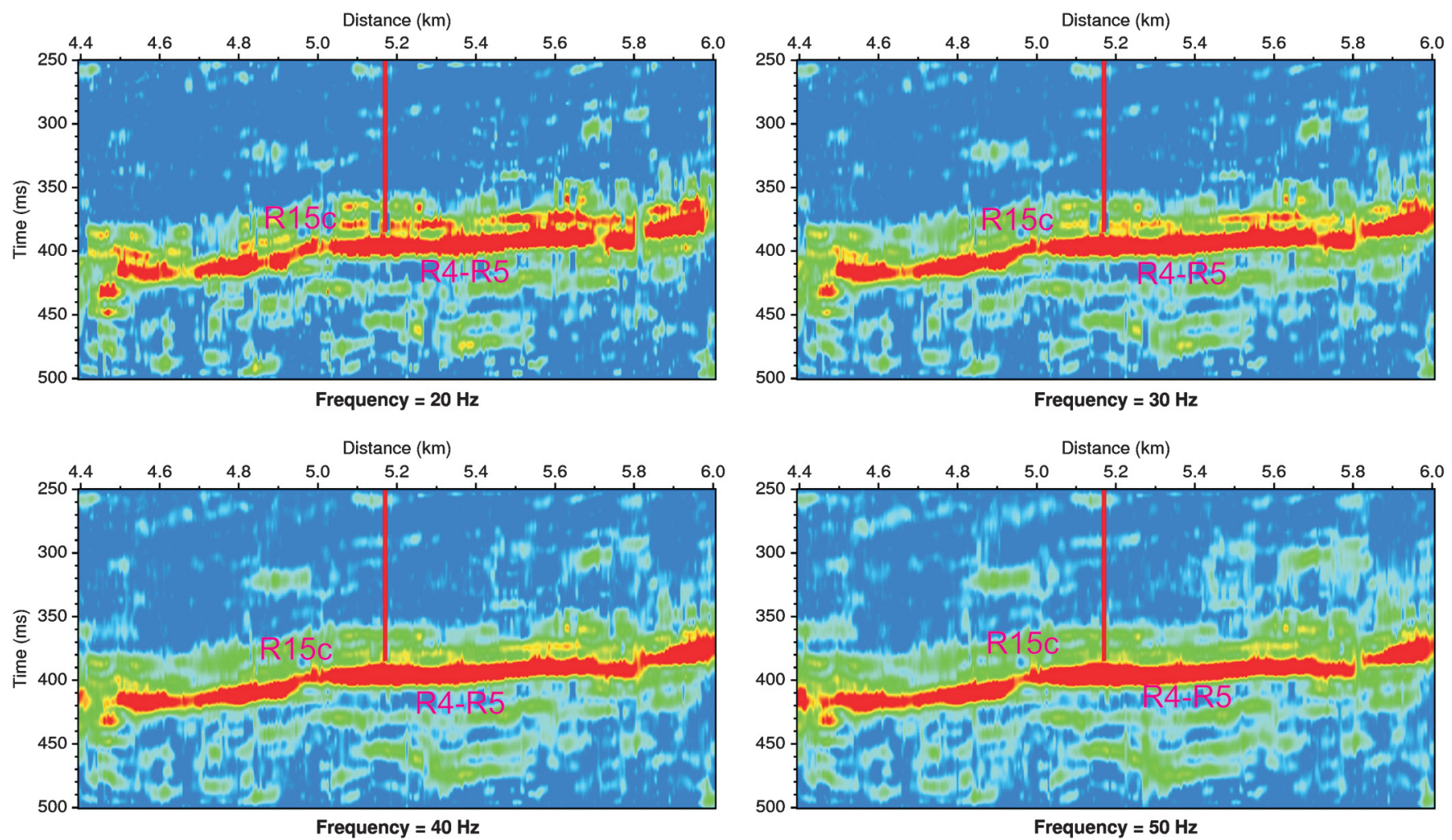

Figure 3. Time-frequency spectra at frequencies $20,30,40$, and $50 \mathrm{~Hz}$, between distance 4.4 and $6 \mathrm{~km}$ along seismic profile W-E 500 . Reflections R4-R5 show strong amplitude in the frequency domain, whereas the target coal-seam reflection R15c has much weak amplitude than R4-R5. 
weaker. Based on the weak amplitude spectrum in the frequency domain, it is difficult to make a direct analysis on the lateral heterogeneity in the coal bed. Thus, such analysis cannot rely on the amplitude information either in the time or frequency domain.

It is possible to use matching pursuit to remove the strong reflection, as suggested by Wang (2010), to analyze the target weak reflection left over in the time domain. However, we will see in the next section that if exploiting the difference in the frequency variation patterns at different time-space locations, the strong reflection in the frequency domain will no longer affect our analysis on the target coal seam immediately above it.

Among many geodynamic parameters, frequency is a sensitive parameter responding to the anomalies within the heterogeneous media. A coal seam should have certain resonant frequency, differing from the natural frequency of surrounding layers. When seismic waves propagate through a coalbed with rich $\mathrm{CBM}$, the resonant frequency will significantly differ from the rest. The resonance changes not only the amplitude (or power) of seismic waveforms, but most importantly the spectral variation pattern in terms of characters in amplitude, phase, and attenuation, etc., in the frequency domain. Therefore, based on the time-frequency spectrum, one can analyze variation pattern to highlight the anomalies in a $\mathrm{CBM}$ reservoir.

So, the essential task here is to generate high-resolution and highfidelity "data" (i.e., the time-frequency spectrum), so that the spectral variation patterns can truly reflect the existence and spatial variation of potential reservoirs. In the following step, I will conduct

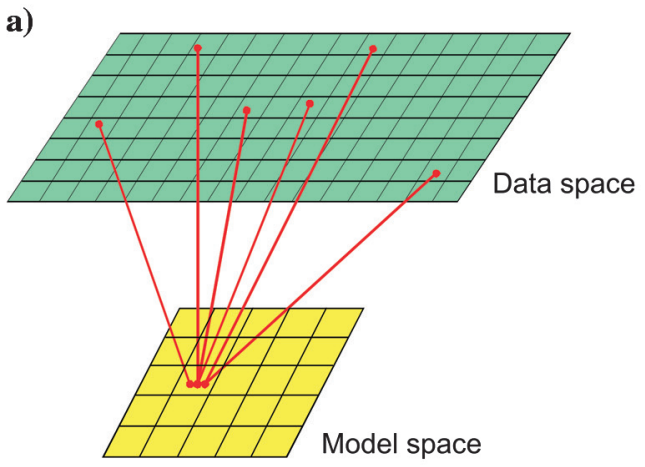

b)

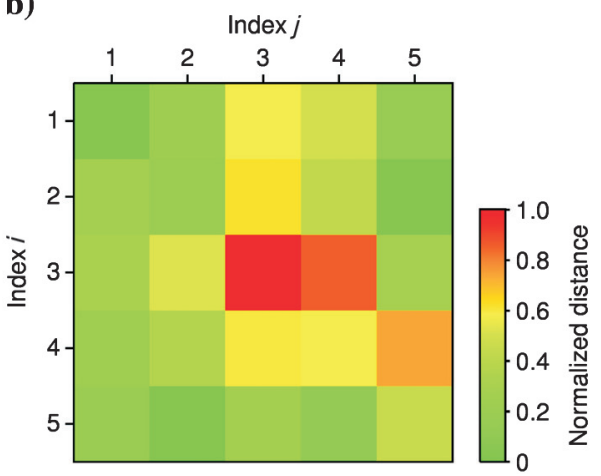

c)

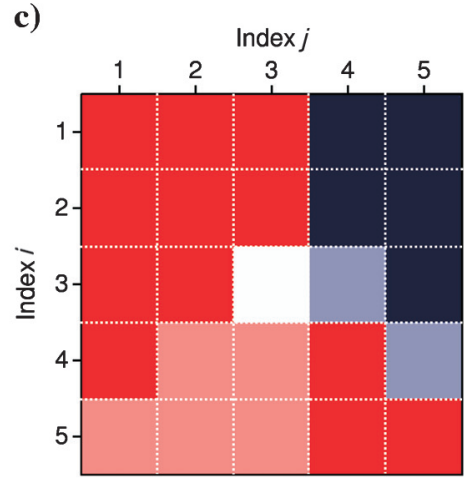

Figure 4. (a) Unsupervised neural network SOM, mapping data (spectra) vectors into the model space on which each weight vector is a representation of a group of data vectors. (b) The distance matrix of weight vectors (neurons) in the model space. (c) Classifying weight vectors (neurons) of the model space into five clusters. clustering analysis to classify these time-frequency spectra into different variation patterns for the purpose of reservoir characterization. In other words, this section raises an extra dimension (frequency) from the original time-space domain, and the next section will be a dimension-reduction process which projects the timespace-frequency data back to the time-space domain.

\section{CLUSTERING ANALYSIS}

For the clustering analysis, I apply the following three methods consecutively. First, I use an unsupervised neural networking method to project the data (spectrum vectors) in the time-space domain onto weight vectors in the model space. Then, I use a hierarchical clustering and a partitioning method in sequence to classify the weight vectors into different clusters in the model space.

\section{The unsupervised neural networking method}

The unsupervised neural networking method I use is the selforganizing map (SOM) algorithm. Although it realizes the nonlinear dimension-reduction process on complicated high-dimensional data, it also can keep the topography of the data structure (Kohonen, 1995). Its abstractive and visualization properties mean that it is well suited to identifying anomalies from a complicated data volume.

SOM attempts to search for representative "models" that can better describe the property of the data (Figure 4a). These representative models are weight vectors, listed in order in 2D space: Similar models are close to each other, and nonsimilar models sit apart. Therefore, the result of SOM is an image of similarity, or clustering image.

The structure of SOM is fairly simple, consisting of only the input layer and the output layer. Data vectors (nodes) in the input layer are linked directly to different weight vectors (neurons) in the output layer. It is a nonparameterized, iterative, and recursive process. Initially, I set randomly the weight vectors (with the same length as a data vector). Then, for any training data vector, neurons compete against each other to find a winner (who is the closest to the input data vector). Only the winner and its neighbors are allowed to further adjust the weights. Eventually, adjusting the weight vectors in the model space will keep the topographic structure of the original data.

A neuron's position in the model space is measured by its distance to neighboring neurons (Ultsh et al., 1993; Kohonen, 1995). Denote the Euclidean distance between two neurons $m(i, j-1)$ and $m(i, j)$ by $u\left(m_{i, j-1}, m_{i, j}\right)$. For a single neuron $m(i, j)$ at index $(i, j)$, the "distance measurement" $d(i, j)$ is the average (or median, maximum, or minimum) of the Euclidean distances $u\left(m_{i \pm 1, j \pm 1}, m_{i, j}\right), \quad u\left(m_{i \pm 1, j}, m_{i, j}\right), \quad$ and $u\left(m_{i, j \pm 1}, m_{i, j}\right)$ between the given neuron $m(i, j)$ and the eight surrounding neighbors. For a matrix of neurons in the model space, the corresponding distance matrix visualizes the distance between neurons (or weight vectors). As 
shown in Figure 4b, the green color corresponds to a small distance between weight vectors, and red indicates a large distance.

The basic idea when using SOM for reservoir characterization is based on the sample's similarity principle. That is, similar data vectors will be projected very closely in the model space, and nonsimilar vectors will sit apart. Therefore, SOM can effectively and efficiently identify and highlight the existence of anomalies. This is a remarkable advantage for analyzing complicated, highdimensional data, in contrast to, for example, a statistical method.

\section{Hierarchical clustering and partitioning}

Seismic frequency spectrum vectors in the time-space domain are linked to the weight vectors (neurons) in the model space. To identify anomalies in the time-space domain, I conduct clustering analysis on these neurons. I jointly use the two conventional clustering methods of hierarchical clustering and partitioning. The hierarchical clustering method I use is the agglomerative algorithm. The partitioning method I use is the $K$-means algorithm.

When running the hierarchical clustering algorithm, for $n$ neuron vectors, it first constructs a $n \times n$ distance matrix, and then clusters these vectors based on distances:

1) defining each vector as an individual cluster

2) finding pair of vectors close to each other and put them into a single cluster

3) calculating the distances between this new cluster and all of the other clusters

4) repeating steps 2 and 3, and updating iteratively

Note that hierarchical clustering is irreversible. Once a vector is classified to a cluster, it cannot be reclassified to another.

The $K$-means algorithm is a simple, but time-consuming, iterative procedure. It divides $n$ neuron vectors into $K$ clusters, each of which has its own center point. In each cluster, the total distance of every vector from the central vector is minimal. The implementation consists of the following steps:

1) selecting $K$ points in the space as the initial central points of $K$ clusters

2) clustering all vectors into these $K$ clusters based on the distance

3) recalculating the central position of each cluster

4) repeat steps 2 and 3, until all central positions have no further shift

This implementation clearly shows shortcomings. The positions of the initial $K$ points are selected arbitrarily, without a standard rule. The final result depends on the selection of the initial $K$ points, and thus is often suboptimal. To obtain an optimal position, one may need to repeat the experiments with different selections of the initial $K$ points, and compare the final results. Sometimes, there exists a null cluster in which all vectors are null. The final result also depends on $K$, the number of clusters set at the beginning, but one often does not know exactly how many clusters are needed for a real data set. The uncertainty of the selection of $K$ value will directly affect the clustering result and, in turn, the final interpretation.

The hierarchical clustering clearly overcomes the problems with the $K$-means method. Therefore, I exploit the respective advantages of these two methods. I first adopt the hierarchical clustering algorithm to cluster the weighting vectors step by step, and then use the
$K$-means algorithm to further adjust the cluster central positions and classification of each vector.

Figure $4 \mathrm{c}$ displays the clustering result of neurons in the model space. Any data vectors which are linked to neurons (weighting vectors) with the same color means they are similar to each other. Otherwise, the data vectors can show a significant difference.

The clustering analysis in the prediction process implicitly takes into account various factors (such as elasticity, viscosity, cleat system, wave interference within coalbed, etc.) in a fairly general sense. To understand the physical mechanism, one needs either numerical or physical modeling data for analysis. However, how to parameterize a CBM model properly and clearly remains a challenging research topic at the current stage.

\section{RESERVOIR CHARACTERIZATION}

The procedure for predicting CBM distribution can be outlined as the following:

1) picking reflection times of a reference event (underneath the target coalbed), and flattening the reference event

2) calculating the time-frequency spectrum of each sampling point over the entire time-space window

3) clustering analysis, as described in the previous section

4) scaling all available CBM information in the study area into eight levels

5) finding an integer value for each clustered spectral variation pattern, so that the resultant potential index matches these eight-level indexes in an optimal sense

6) finally, assigning determined integer values to all patterns and calculating potential indexes over all samples within the timespace window

The potential index is a sum of pattern values over a time interval for each trace, and is then normalized over space to match the eightlevel CBM gas production.

First, I pick the combined reflection "R4-R5" (the bottom of formation $\mathrm{T}$ in Upper Carboniferous and the top of Ordovician limestone) merely because its strength makes it easily identifiable, for use as a reference. I take a time window starting from $90 \mathrm{~ms}$ above the reference time and ending at $10 \mathrm{~ms}$ below (Figures 5a and 6a). Then, I flatten the windowed profile along the reference time, so that I can clearly see the lateral variation of the relatively weak reflection R15c, at about 75 ms (Figures $5 \mathrm{~b}$ and $6 \mathrm{~b}$ ).

As described in the previous section, for a time-space domain seismic profile, there is an associated data cube with the frequency as the third dimension. This is the basis for our reservoir characterization. The clustering analysis generates a time-space domain image of the patterns of spectral variation along this third axis (Figures $5 c$ and $6 c$ ). In this example, there are five clusters: $a, b$, $\mathrm{c}, \mathrm{d}$, and e. The features of spectral variations around coal seam 15 differ from that of surrounding rocks. Even for a strong reflection such as R4-R5, the strength no longer has any influence. Only the spectral variation pattern plays a role here. This is the most significant feature of this technology. In fact, the strong variation at the top of the time window (within 0-30 ms) also reflects the existence of coal seam 3 above.

I assign an integer value between one and five to each of the five patterns a, b, c, d, and e. Then I make a sum over the time interval of 50-100 ms at each individual trace, and normalize the sum values over all traces in the space. I divide the normalized value into eight 
CBM levels, calibrating with gas productions in the study area (Figures $5 \mathrm{~d}$ and $6 \mathrm{~d}$ ). For the integer values $1-5$, there are 120 permutations. I find the optimal permutation that has the eight-level quantitative indexes best matching the production data.

There are 23 wells producing gas from CBM reservoirs. The eight levels correspond to the accumulated gas productions in the following scale:

\begin{tabular}{ccccccc} 
Index & 0 & 1 & 2 & $\ldots$ & 7 & 8 \\
Production $\left(\times 10^{4} \mathrm{~m}^{3}\right)$ & $<1$ & $1-1.9$ & $2-2.9$ & $\ldots$ & $7-7.9$ & $>8$ \\
\hline
\end{tabular}

a)
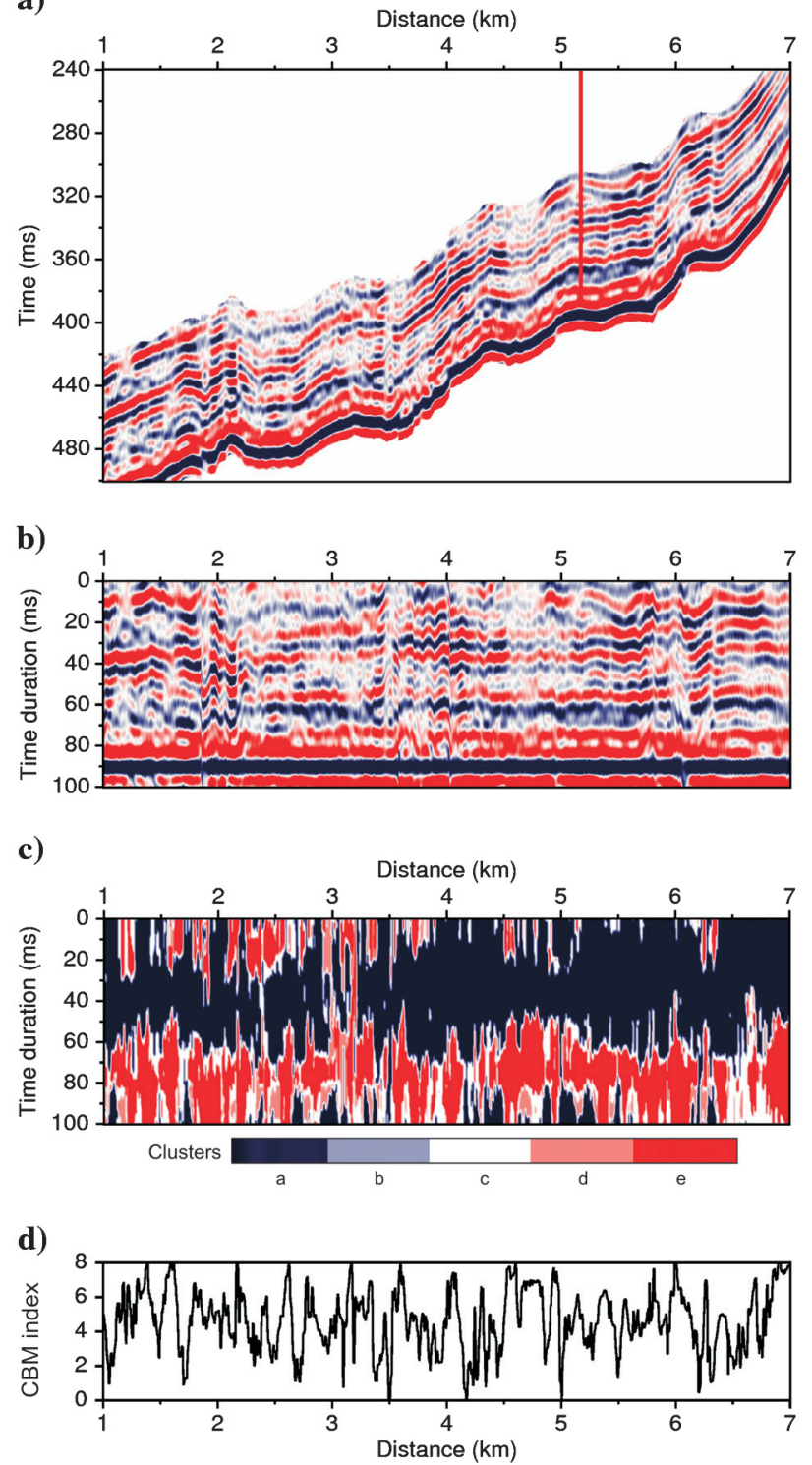

Figure 5. CBM prediction along seismic profile W-E 500: (a) Picking the strong reflection (the combined reflection R4-R5, the bottom of formation $\mathrm{T}$ in Upper Carboniferous and the top of Ordovician limestone) as a reference, (b) flattening the profile along the reference, (c) clustering analysis, and (d) a quantitative indication of lateral variation along coalbed 15 .
I adopt a "take-one-out" strategy to verify the reliability of the method. That is, I use 22 wells out of 23 in the calibration, and then test prediction against the unused well.

Figures 5 and 6 are the prediction of CBM distribution along coal seam 15 . The same procedure is applied to coal seam 3 as well. In both cases, I do not take out the influence of the coal seam thickness variation because the thickness is assumed to be a constant average relatively in this area.

Predicted potential index values are smoothed over the entire study area. Finally, I obtain the spatial CBM distribution along coal seam 15 and 3, as shown in Figure 7. As expected, coal seam 15 has more potential than coal seam 3 . The latter hardly has any index value more than four. The positions with index $\geq 4$ in coal seam 15 are the most prospective CBM locations.
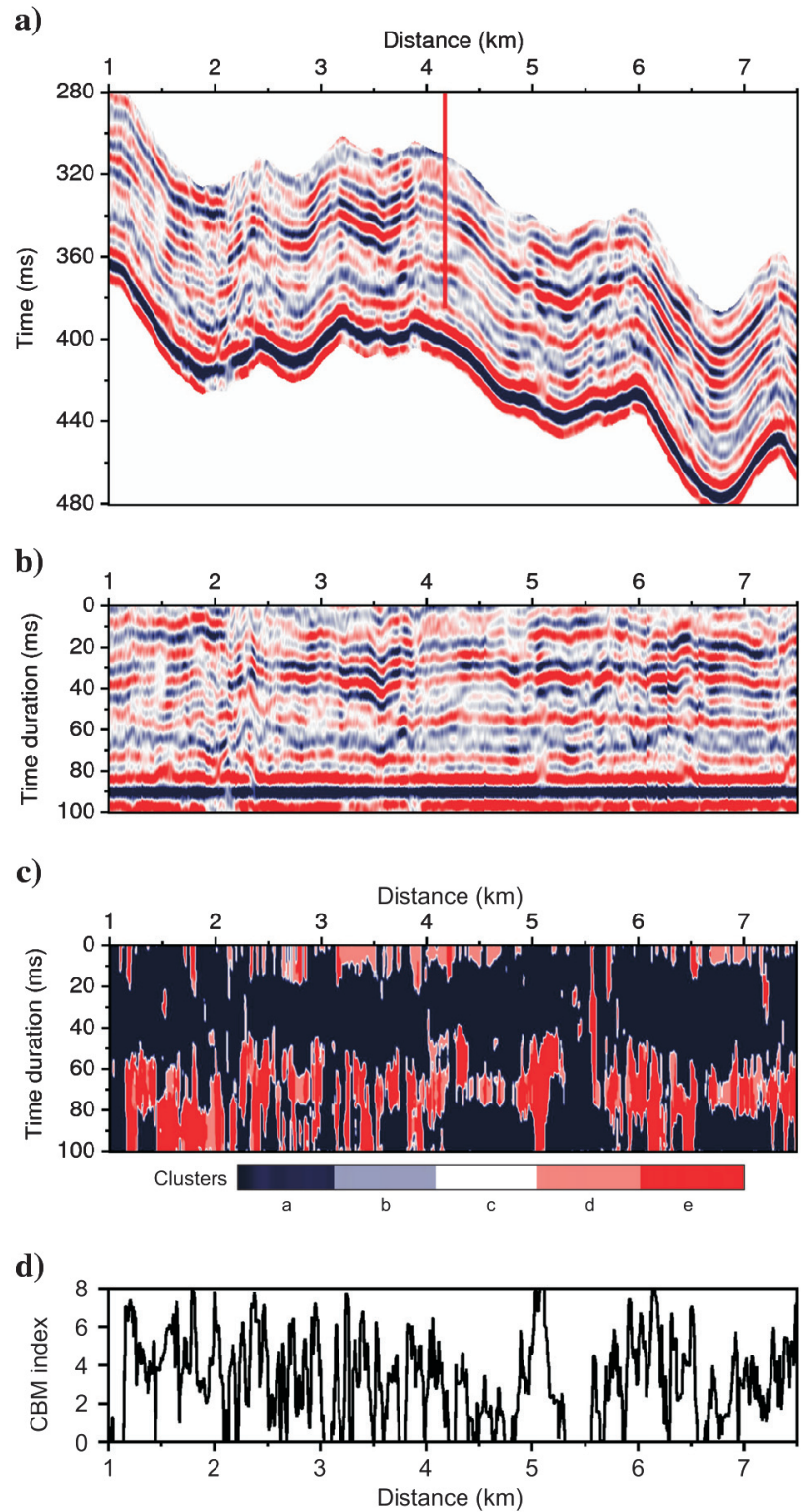

Figure 6. CBM prediction along seismic profile S-N 500: (a) Picking the strong reflection R4-R5 as a reference, (b) flattening the profile along the reference, (c) clustering analysis, and (d) a quantitative indication of lateral variation along coalbed 15 . 


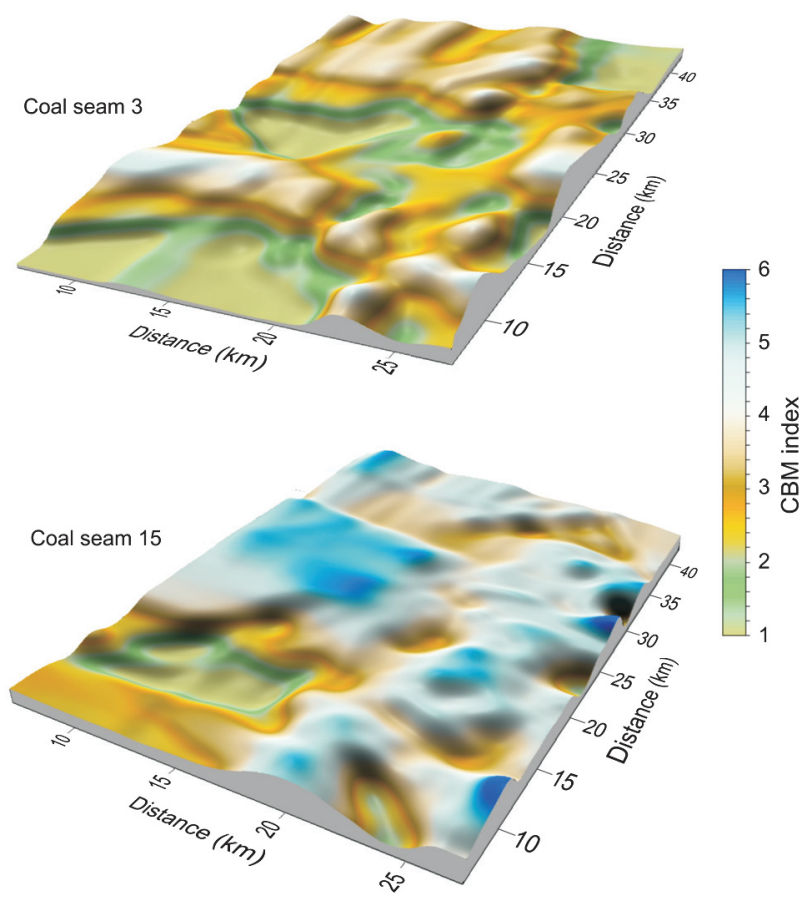

Figure 7. Spatial distribution of CBM reservoirs along coal seam 15 and coal seam 3 .

\section{CONCLUSIONS}

The patterns of spectral variation in the frequency dimension provide a useful source of information for reservoir characterization. The procedure consists of three steps. First, I generate a timefrequency spectrum for every sampling point of a time-space domain seismic profile, raising an extra dimension: the frequency. Then, I conduct a clustering analysis based on the spectral variation patterns. The result is an image back in the time-space domain. Finally, I calibrate visualized anomalies with the CBM production in the study area, and predict its spatial distribution over the target horizons. Although alternative methods to each of these three steps can be adopted, the essential concept presented here is to exploit spectral variation features for reservoir characterization. One potential application of this technique is for the analysis of "weak signals" with low amplitudes because this method is based on the spectral variation along the frequency axis rather than on the signal strength either in the time or frequency domain.

\section{ACKNOWLEDGMENTS}

The author is grateful to the sponsors of the Centre for Reservoir Geophysics, Imperial College London, for supporting this research.

\section{REFERENCES}

Bachu, S., and K. Michael, 2003, Possible controls of hydrogeological and stress regimes on the producibility of coalbed methane in Upper Cretaceous-Tertiary strata of the Alberta basin, Canada: AAPG Bulletin, 87, 1729-1754, doi: 10.1306/06030302015.

Kohonen, T., 1995, Self-organizing maps: Springer.

Mallat, S., and Z. Zhang, 1993, Matching pursuit with time-frequency dictionaries: IEEE Transactions on Signal Processing, 41, 3397-3415, doi $10.1109 / 78.258082$

Mallat, S. G., 2009, A wavelet tour of signal processing: Academic Press.

Morlet, J., G. Arens, E. Fourgeau, and D. Giard, 1982a, Wave propagation and sampling theory - Part I, complex signal and scattering in multilayered media: Geophysics, 47, 203-221, doi: 10.1190/1.1441328.

Morlet, J., G. Arens, E. Fourgeau, and D. Giard, 1982b, Wave propagation and sampling theory - Part II, sampling theory and complex waves: Geophysics, 47, 222-236, doi: 10.1190/1.1441329.

Peng, S. P., H. Chen, R. Z. Yang, Y. F. Gao, and X. P. Chen, 2006, Factors facilitating or limiting the use of AVO for coalbed methane: Geophysics, 71, no. 4, C49-C56, doi: 10.1190/1.2217137.

Shuck, E. L., T. L. Davis, and R. D. Benson, 1996, Multicomponent 3D characterization of a coalbed methane reservoir: Geophysics, 61, 315330. doi: $10.1190 / 1.1443961$.

Ultsh, A., 1993, Self-organizing neural networks for visualization and classification, in O. Opitz, B. Lausen, and R. Klar, eds., Information and classification, concepts, methods and applications: Springer-Verlag, 307-313

Wang, Y., 2007, Seismic time-frequency spectral decomposition by matching pursuit: Geophysics, 72, no. 1, V13-V20, doi: 10.1190/1.2387109.

Wang, Y., 2010, Multichannel matching pursuit for seismic trace decomposition: Geophysics, 75, no. 4, V61-V66, doi: 10.1190/1.3462015. 